\title{
Daytime soybean transcriptome fluctuations during water deficit stress
}

\author{
Fabiana Aparecida Rodrigues ${ }^{1}$, Renata Fuganti-Pagliarini ${ }^{1}$, Juliana Marcolino-Gomes ${ }^{1,2}$, Thiago Jonas Nakayama ${ }^{1,3}$, \\ Hugo Bruno Correa Molinari ${ }^{4,8}$, Francisco Pereira Lobo ${ }^{5}$, Frank G Harmon ${ }^{6,7}$ and Alexandre Lima Nepomuceno ${ }^{1,8^{*}}$
}

\begin{abstract}
Background: Since drought can seriously affect plant growth and development and little is known about how the oscillations of gene expression during the drought stress-acclimation response in soybean is affected, we applied Illumina technology to sequence 36 cDNA libraries synthesized from control and drought-stressed soybean plants to verify the dynamic changes in gene expression during a 24-h time course. Cycling variables were measured from the expression data to determine the putative circadian rhythm regulation of gene expression.

Results: We identified 4866 genes differentially expressed in soybean plants in response to water deficit. Of these genes, 3715 were differentially expressed during the light period, from which approximately $9.55 \%$ were observed in both light and darkness. We found 887 genes that were either up- or down-regulated in different periods of the day. Of 54,175 predicted soybean genes, $35.52 \%$ exhibited expression oscillations in a $24 \mathrm{~h}$ period. This number increased to $39.23 \%$ when plants were submitted to water deficit. Major differences in gene expression were observed in the control plants from late day (ZT16) until predawn (ZT20) periods, indicating that gene expression oscillates during the course of $24 \mathrm{~h}$ in normal development. Under water deficit, dissimilarity increased in all time-periods, indicating that the applied stress influenced gene expression. Such differences in plants under stress were primarily observed in ZTO (early morning) to ZT8 (late day) and also from ZT4 to ZT12. Stress-related pathways were triggered in response to water deficit primarily during midday, when more genes were up-regulated compared to early morning. Additionally, genes known to be involved in secondary metabolism and hormone signaling were also expressed in the dark period.
\end{abstract}

Conclusions: Gene expression networks can be dynamically shaped to acclimate plant metabolism under environmental stressful conditions. We have identified putative cycling genes that are expressed in soybean leaves under normal developmental conditions and genes whose expression oscillates under conditions of water deficit. These results suggest that time of day, as well as light and temperature oscillations that occur considerably affect the regulation of water deficit stress response in soybean plants.

Keywords: Abiotic stress, Daily oscillation, diel regulation, Drought, Glycine max, Plant metabolism

\section{Background}

Soybean is one of the most important crops in the world. Overall yield is highly affected by water deficit stress, particularly when the stress occurs during flowering and early pod expansion [1]. To overcome the water limitation and facilitate the continued expansion of soybean productivity

\footnotetext{
* Correspondence: alexandre.nepomuceno@embrapa.br

'Brazilian Agricultural Research Corporation- Embrapa Soybean, Embrapa

Soybean- Rod. Carlos João Strass, s/n, Londrina 86001-970PR, Brazil

${ }^{8}$ Embrapa LABEX US Plant Biotechnology at ARS/USDA Plant Gene

Expression Center, Albany, CA, USA

Full list of author information is available at the end of the article
}

and crop improvement, implementation of modern biotechnology such as genetic engineering of plants to produce drought-tolerant cultivars, rises as a potential solution [2]. However, the achievement of such a goal is highly dependent on the elucidation of the molecular mechanisms of drought tolerance and their interaction with environmental cues. A better understanding of these aspects would help identify candidate genes for genetic engineering of improved stress-tolerant crops [3].

To better fit to the surrounding environmental conditions, such as season and light/darkness and temperature variations, it is known that plants, as sessile organisms, 
coordinate and regulate their metabolism and physiology through an endogenous circadian clock. This clock drives rhythms at the molecular and cellular levels and, thus, temporally regulates plant physiology and behavior to anticipate changes in the environment [4]. According to Khan et al. [5], the consequence of proper clock and environment synchronization is optimized fitness. However, abiotic stresses, such as drought, change the clock synchrony, altering the circadian rhythm in response to dehydration [6].

Genome-wide analysis of mRNA expression shows daily oscillation coordinated by circadian rhythms, which, in turn, regulate various biological processes [7-9], such as seed dormancy and germination [10], hormone metabolism [11-13], and grapevine fruit ripening [14], among other processes [10]. Gene expression regulated by the circadian rhythm also has been observed in some plant responses to abiotic stress [15-17]. In the process of cold acclimation in Arabidopsis, gene expression was affected by time of day, revealing an interaction between cold and diurnal regulation that drives transcriptome changes [15]. Furthermore, time of day also was an important cue for triggering changes in the Populus transcriptome [16] and in Arabidopsis plants in response to soil drying [17]. In soybean, evidence also suggests that the circadian rhythm plays a role in regulating genes involved in developing seeds [18].

Although soybean is one of the most studied crops using molecular biology tools, little is known about how daily oscillations of gene expression are affected by drought stress during the survival or acclimation response. The regulation of a proline-rich-protein gene, induced under drought and salt stresses in specific tissues of soybean seedlings, was demonstrated to be circadiancontrolled [19]. Considering the dynamic changes of plant metabolism that occur to coordinate the daily variation in light and temperature, the evaluation of gene expression at different time periods of the day becomes valuable for identifying times during which key genes might be most influential in the defense response [17]. In Arabidospis, hormone-related genes, specifically the abscisic acid (ABA)-responsive genes, are correlated with diurnal oscillations [12]. The functional roles of some representatives, like the dehydrins class and the $r d 29 A$, or the cold-regulated COR15B/15A and the low temperatureinduced LTI30, may be related to responses to water deficit and low temperature, respectively [12].

Recently, our research team has demonstrated how drought impacts diurnal oscillation of both droughtresponsive and circadian clock genes in soybean [20]. Drought stress induced marked reduction in gene expression levels of several circadian clock-like components, such as GmLCL1-, GmELF4-, and GmPRR-like genes. The same conditions produced a phase advance of expression for the GmTOC1-, GmLUX- and GmPRR7like genes. Similarly, the daily oscillation pattern of the soybean drought-responsive genes DREB-, bZIP-, GOLS-, $R A B 18$ - and Remorin-like changed significantly following plant exposure to water deficit.

With the goal of detecting genome-wide transcriptome changes during the entire period that plants were exposed to moderate water deficit and if such variations occurred in a time-of-day-dependent manner, we analyzed multiple time points in a diel period. Here, we present a survey of soybean genes expressed under stress and their daily oscillation waveforms during a 24-h time course. We also determined their abundance, and we suggest the putative biological roles of these differentially expressed genes.

\section{Results}

Genes differentially expressed in response to water deficit The expression pattern of soybean genotype BR16, previously characterized as drought sensitive [21], was evaluated under normal and water deficit conditions, during a 24-h time course. To identify differentially expressed genes (DEGs) in response to water deficit treatment, we applied a stringent statistical test to determine whether genes were either up- or down-regulated compared to those of plants under optimal hydration conditions. The resulting ratio represented the fold-change $(\mathrm{fc})$ for each gene. To avoid false positives and reliably identify the most significant changes in gene expression, only genes with $\mathrm{fc} \leq-2$ (down) and $\geq 2$ (up) were considered. We also applied a stringent statistical significance cutoff (adjusted $p$-value $\leq 0.01$ ) to improve confidence (Additional file 1).

There were larger sets of differentially expressed genes in ZT0 $(n=2218)$ and ZT4 $(n=1290)$ compared to the other periods. In ZT0, the majority of genes (73.76 \%) were down-regulated under water deficit stress condition, this is in contrast to the other periods (ZT4, ZT8, ZT12, and ZT20), in which genes were primarily upregulated $(66.35 \%, 84.54 \%, 90.14 \%$, and $56.99 \%$, respectively) (Fig. 1, Additional file 1). In general, a moderate expression ratio was detected for most genes, but high fold-changes for some genes were observed in the ZT0 and ZT4 periods. The highest differential expression was detected in ZT0 for Glyma18g43980 (156.41 fc), which codes for a UDP-glucosyl transferase 73B5, and for Glyma10g38050 (134.53 fc), a CAP160 protein. In ZT4, the highest confidence levels were found for Glyma03g24320, a gene related to the fatty acid hydroxylase superfamily and for Glyma17g13720, with unknown function (Fig. 1, Additional file 1).

In this study, we found 4866 genes that were differentially expressed in soybean plants in response to moderate water deficit during a 24-h time course (Fig. 1, 

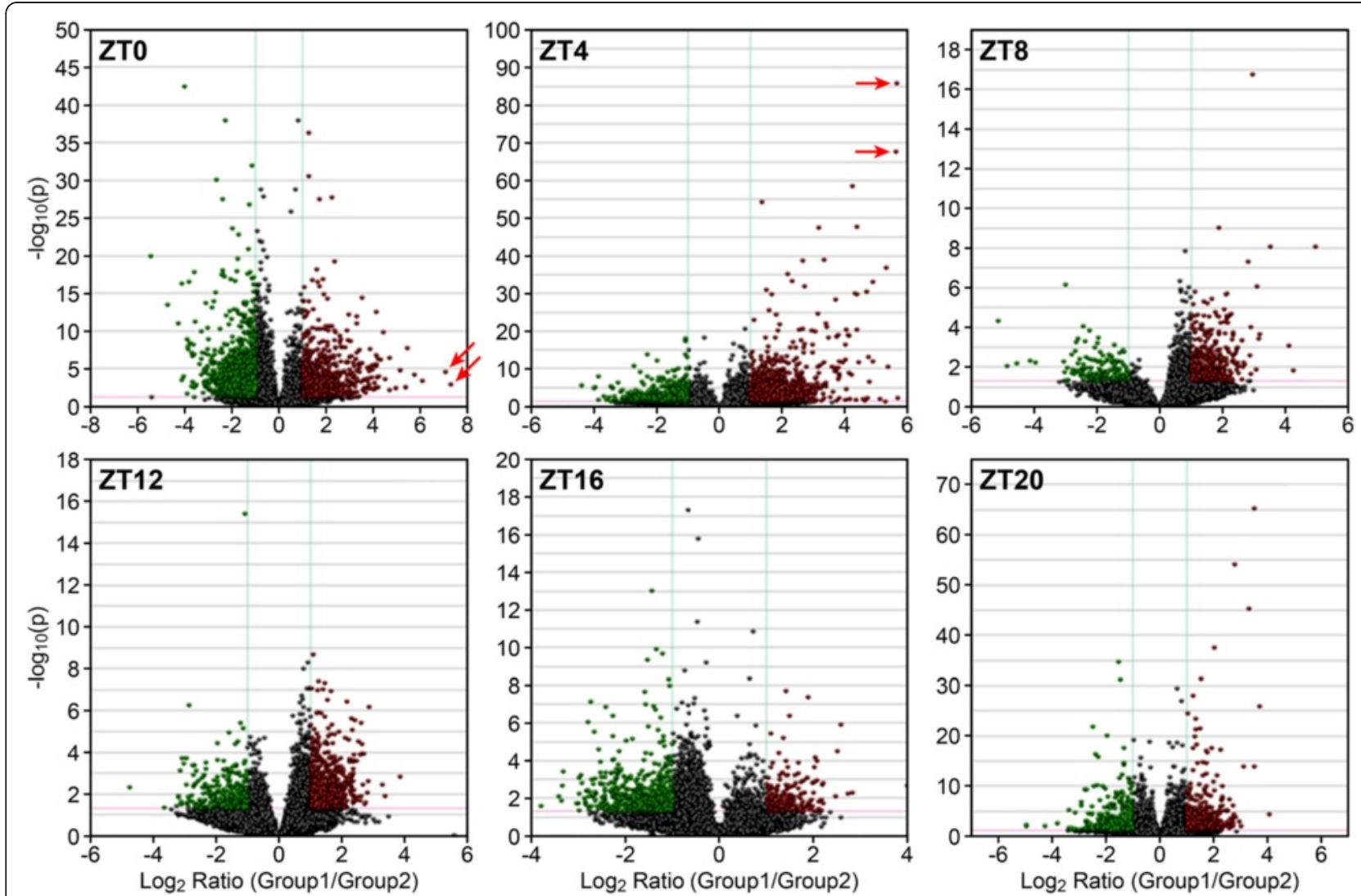

Fig. 1 Volcano plots. Expression data were plotted on a $\log _{2}$ scale ( $x$-axis) versus a - $\log _{10}$ transformation of the $p$-value ( $y$-axis). Datasets were filtered to remove genes with low expression levels (blue lines from - 1 to 1 on the $x$-axis), and a significance cut off ( $p<0.01)$ was applied (red line on the y-axis). ZTO ( $n=2218 ; 582$ up and 1636 down); ZT4 ( $n=1290 ; 856$ up and 434 down); ZT8 ( $n=207 ; 175$ up and 32 down); ZT12 ( $n=497 ; 448$ up and 49 down); ZT16 ( $n=175 ; 73$ up and 102 down); and ZT20 ( $n=479 ; 273$ up and 206 down). Arrows indicate the highest expression in ZTO (Glyma18g43980 and Glyma10g38050) and the highest confidence in ZT4 (Glyma03g24320 and Glyma17g13720)

Additional file 1). These genes represented $8.98 \%$ of the 54,175 predicted genes in the Glyma 1.1 soybean genome assembly [22]. However, considering that some genes ( $n=887$; Fig. 2 ) were either up- or down- regulated during multiple time periods, 3979 genes (approximately $7.34 \%$ of the genome) were uniquely expressed in response to water deficit. The number of genes exclusively expressed in each time period and those observed in multiple ones are presented in Fig. 2 and in Additional file 2. Most of the DEGs were identified in the early and late morning periods (ZT0 and ZT4). Approximately $72.2 \%$ (ZT0; 279 genes up-regulated and 1323 genes downregulated) and 53 \% (ZT4; 502 genes up- and 182 genes down-) were expressed exclusively in one period, whereas others were detected in both periods (ZT0-ZT4; $n=399$ ) (Fig. 2, Additional files 1 and 2). Fewer genes were differentially expressed in ZT8 ( $n=207 ; 175$ genes up- and 32 genes down-) and ZT16 ( $n=175 ; 73$ genes up- and 102 genes down-) in response to water deficit (Additional file 1). However, most of these genes were exclusively detected in ZT8 (87.9 \%; 155 genes up- and 27 genes down-)

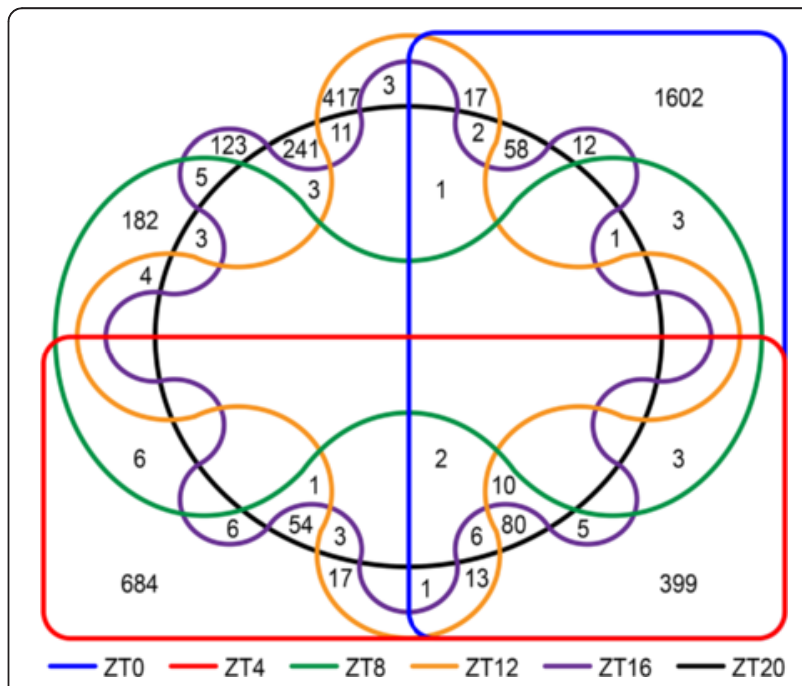

Fig. 2 Edwards's diagram. Number of genes that were differentially expressed in each time period or in more than one period. ZTO $(n=2218) ;$ ZT4 $(n=1290) ; Z T 8(n=207) ; Z T 12(n=497) ; Z T 16$ $(n=175)$; and ZT20 $(n=479)$ 
and ZT16 (70 \%; 53 genes up- and 70 genes down-), respectively (Fig. 2, Additional file 2). Similarly, in ZT12 (n $=497 ; 448$ genes up- and 49 genes down-) (Additional file 1), $83.9 \%$ of the genes (379 up- and 38 down-) were found only in this time period (Fig. 2, Additional file 2). At predawn (ZT20), 479 genes (273 up- and 206 down-) were identified as differentially expressed (Additional file 1), from which 144 up- $(52.7 \%)$ and 97 down-regulated (47\%) genes were observed exclusively in this time period (Fig. 2, Additional file 2).

In general, genes differentially expressed in ZT0-ZT4 kept their expression profiles during both time periods, either increasing (Glyma01g41330 [coding for expansinlike B1], Glyma12g10670 [Ras-related small GTP-binding family protein], and Glyma16g02390 [homeobox 7]) or decreasing (Glyma08g10435, Glyma08g10440, Glyma13g31410 [aluminium-induced protein with YGL and LRDR motifs], and Glyma15g08300 [dormancy-associated protein-like 1]) their differential expression levels from early to late morning (Fig. 3). Three upregulated genes that were detected during morning (ZT0-ZT4) also exhibited decreased differential expression (Glyma17g10950 [expansin A15]) or changed their profiles (Glyma07g31380 [cytochrome P450] and Glyma01g34236) at the late day (ZT8) (Fig. 3). We found 58 genes identified as differentially expressed in both ZT0 (early morning) and ZT20 (dark period) (Fig. 2). Dynamic changes in expression were not observed for these genes, with the exception of Glyma11g33040, which codes for an oxidative stress 3 protein that was upregulated in ZT0 but down-regulated in ZT20 (Fig. 3).

Eighty other genes were expressed in early and late morning (ZT0-ZT4) and again in ZT20. Equivalent gene expression profiles were observed at these time periods, as exemplified by Glyma03g37140 (outer membrane tryptophan-rich sensory protein) (TSPO)related, Glyma05g20400 (amino acid kinase family protein), Glyma06g02380 (DC1 domain-containing protein), Glyma06g45043 (O-methyltransferase 1), Glyma06g45046, Glyma06g45050, Glyma14g32430 (highly ABA-induced PP2C gene 3), and Glyma17g13720, whose expression peaks reached their highest induced levels in ZT4 (Fig. 3). Alternatively, Glyma15g40070, which codes for a 9-cisepoxycarotenoid dioxygenase 3 (NCED3) enzyme, exhibited a decreasing expression pattern between the light and dark periods (Fig. 3), although it maintained its upregulated profile. Glyma17g14830 (nitrate transporter 1.1), Glyma19g34380 (indole-3-acetic-acid-inducible 14), and Glyma02g00760 were up-regulated at midday (ZT4) but down-regulated in ZT16 (Fig. 3).

No genes in common were detected for all time periods; however Glyma08g14670 and Glyma20g29770 were upregulated in five out of the six sampling times, the exception being the ZT8 period (Fig. 2). Interestingly, the expression levels of these two genes were higher in ZT0 and ZT4compared to the other periods (Fig. 3). Similar expression profiles were observed for Glyma01g07390 (an ABI 5-binding protein), Glyma03g24320 (fatty acid hydroxylase superfamily), and Glyma19g11770 (a highly ABAinduced PP2C gene 2) in ZT0-ZT4-ZT16-ZT20 (Fig. 3). During these same time periods, we found seven other genes that were up- or down-regulated. However, no contrasting changes were observed among these periods.

Some genes (Glyma04g04050 [BRI1 kinase inhibitor 1], Glyma02g05050 [eukaryotic aspartyl protease family

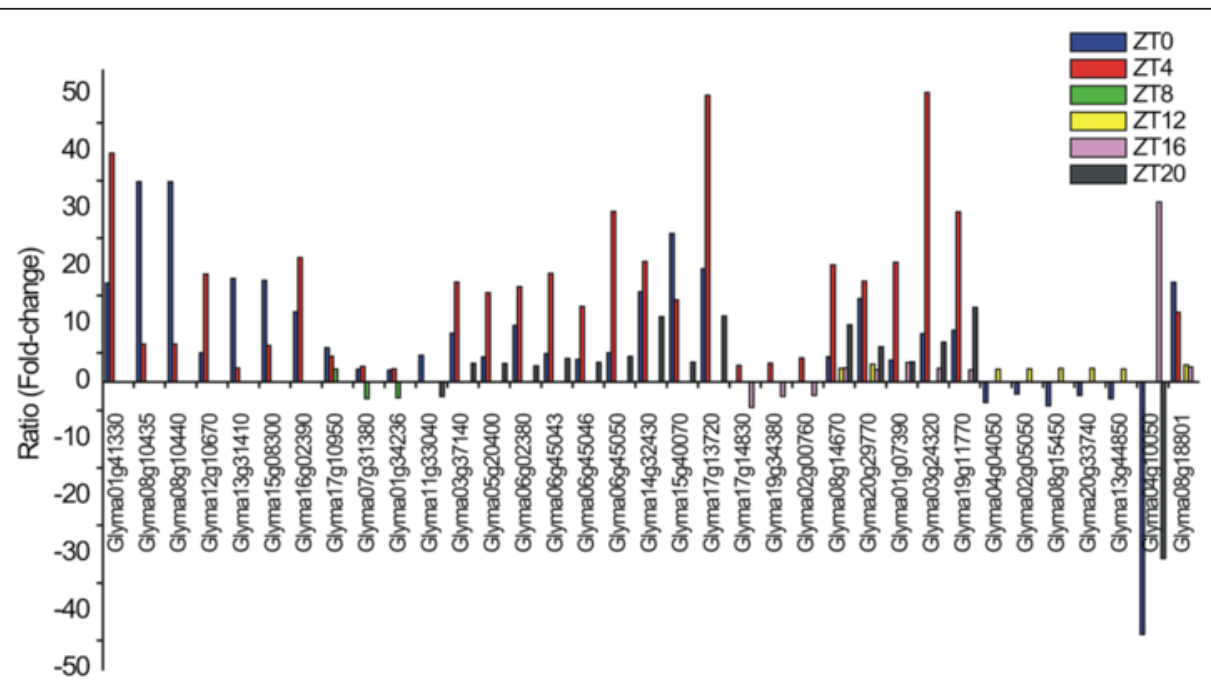

Fig. 3 Genes differentially expressed in different time periods with a diverse expression pattern. Gene expression was analyzed with edgeR statistical test to determine a ratio of expression (fold-change) between control and drought-stressed plants. The $y$-axis represents the fold-change value. All data shown are statistically significant (adjusted $p$-value of $p \leq 0.01$ ) 
protein], Glyma08g15450, Glyma20g33740 [LRR- and NB-ARC-domain-containing disease-resistance protein], and Glyma13g44850 [leucine-rich receptor-like protein kinase family protein]) were down-regulated in ZT0 and up-regulated in ZT12, a transition light-dark period (Fig. 3). Likewise, the transcript Glyma04g10050 (MSCSlike 3) identified in ZT0-ZT8-ZT20 was down-regulated $(-43.94 \mathrm{fc}$; ZT0) in the early morning, up-regulated later during the day ( $31.26 \mathrm{fc}$; ZT8), and down-regulated at the end of the dark period (-30.79 fc; ZT20) (Fig. 3), showing oscillating expression during the day. In addition, an important water-deficit related gene (Glyma08g18801 [9-cisepoxycarotenoid dioxygenase 5] [NCED5]) was dynamically up-regulated over time (ZT0 - ZT4-ZT12 - ZT16), showing higher differential expression levels in ZT0 and ZT4 periods compared to ZT12 and ZT20 (Fig. 3). A list comparing all genes identified as differentially expressed in all time periods is presented in Additional file 2.

\section{Functional roles of differentially expressed soybean genes in response to water deficit}

Gene Ontology (GO) terms were associated with the DEGs to assess their putative biological roles. We performed an enrichment analysis of such terms comparing the list of DEGs identified in each time point with the annotation of the entire soybean genome (Fig. 4, Additional file 3). Initially, we found a set of $165 \mathrm{GO}$ terms enriched among time periods (ZT0 - ZT20), including Biological Process, Molecular Function and Cellular Component terms. Aiming to summarize the GO terms obtained, the resulting lists were analyzed by REVIGO method [23] to remove redundant GO terms. Approximately $50 \%$ of the processes expressed by soybean plants under water deficit were enriched in ZT0. Processes including the regulation of biological processes, transcription, and transcription factor activity were up-regulated in ZT0, whereas, among the down-regulated genes, the enriched processes were primarily represented by translation and metabolic processes (Fig. 4). Likewise, the same processes observed in both down- and up-regulated genes in ZT0, among others, were enriched in ZT4 (Fig. 4). In the ZT8 time period, there were no enriched processes for downregulated genes, but DNA binding and processes related to cellular component organization were significantly represented among up-regulated genes (Fig. 4). Lipid metabolic processes were enriched in ZT12, while translation and structural molecular activity were down-regulated (Fig. 4). In ZT16, no enriched processes were detected for up-regulated genes, and only processes related to cellularcomponent terms were detected among down-regulated genes (Fig. 4). Interestingly, for genes expressed in ZT20 (pre-dawn), we observed that transcription factor activity and DNA metabolism were the only two significant processes enriched in $28.4 \%$ of the annotated genes, the same processes enriched during light periods (ZT0-ZT8). All enriched processes observed during the 24-h time course are described in Additional file 3.

\section{Diel oscillations in the expression of soybean genes under water deficit}

Expression data (reads per kilobase per million [RPKM]) from control plants and those under water deficit were collected separately to determine the putative rhythmicity of gene expression. Rhythmic waveforms were detected for 19,240 and 21,248 genes in control and water deficit plants (false discovery rate [FDR] correction, adjusted $p$-value $<0.05)$, respectively, which correspond to $35.52 \%$ and $39.23 \%$ of the whole genome (Glyma 1.1) (Additional files 4 and 5). As expected, functional classes were similar in both the control and treated plants since they shared the majority of the genes $(n=14,484)$. Most were organized into protein (synthesis, targeting, and degradation; $21.78 \%$ ) and RNA (regulation of transcription; $18.04 \%)$ classes. Few differences were observed in classes including transport and signaling between the control (7.06 \% and $7.02 \%$, respectively) and drought-stressed plants ( $7.79 \%$ and $8.11 \%$, respectively) (Fig. $5 \mathrm{a}$ ).

We also identified groups of genes that showed expression fluctuations exclusively in control $(n=4756)$ or water deficit $(n=6764)$ conditions (Fig. 5b, Additional file 6). Under normal water supply, genes expressed in a time-of-day-dependent manner played a role in several plant biological processes, particularly in the RNA (regulation, transcription, and splicing; $12.73 \%$ ) and protein (16.44 \%) classes. Interestingly, genes associated with stress response $(3.27 \%)$ were detected only in the control plants (Fig. 5b, Additional file 6). Most of those genes are involved in the response to biotic stress $(n=$ $110)$ and heat stress $(n=35)$. Similarly, expression of genes related to redox regulation metabolism (thioredoxin, ascorbate, glutathione, glutaredoxins, dismutase, and catalases; $1.25 \%$ ) was observed in the control plants (Fig. 5b).

The functionality of genes detected only in the water deficit condition was significantly increased (compared to control ones) in signaling (G-proteins, sugar, receptor-kinases, and leucine-rich repeat II; $8.94 \%$ ), RNA (regulation; $17.89 \%$ ), and protein (synthesis, post-translational modification, and degradation; $21.32 \%$ ) categories. Additionally, genes involved in hormone (synthesis/degradation of ABA, brassinosteroid, ethylene, jasmonate, salicylic acid, and hormone-mediated signaling; $3.37 \%$ ), amino acid $(1.44 \%)$, and lipid (degradation and beta oxidation; $2.40 \%)$ metabolism also were more highly represented (Fig. 5b). After subjecting plants to water deficit, genes from the stress response class increased by $4.98 \%$ and were particularly associated with cold $(n=110)$, 


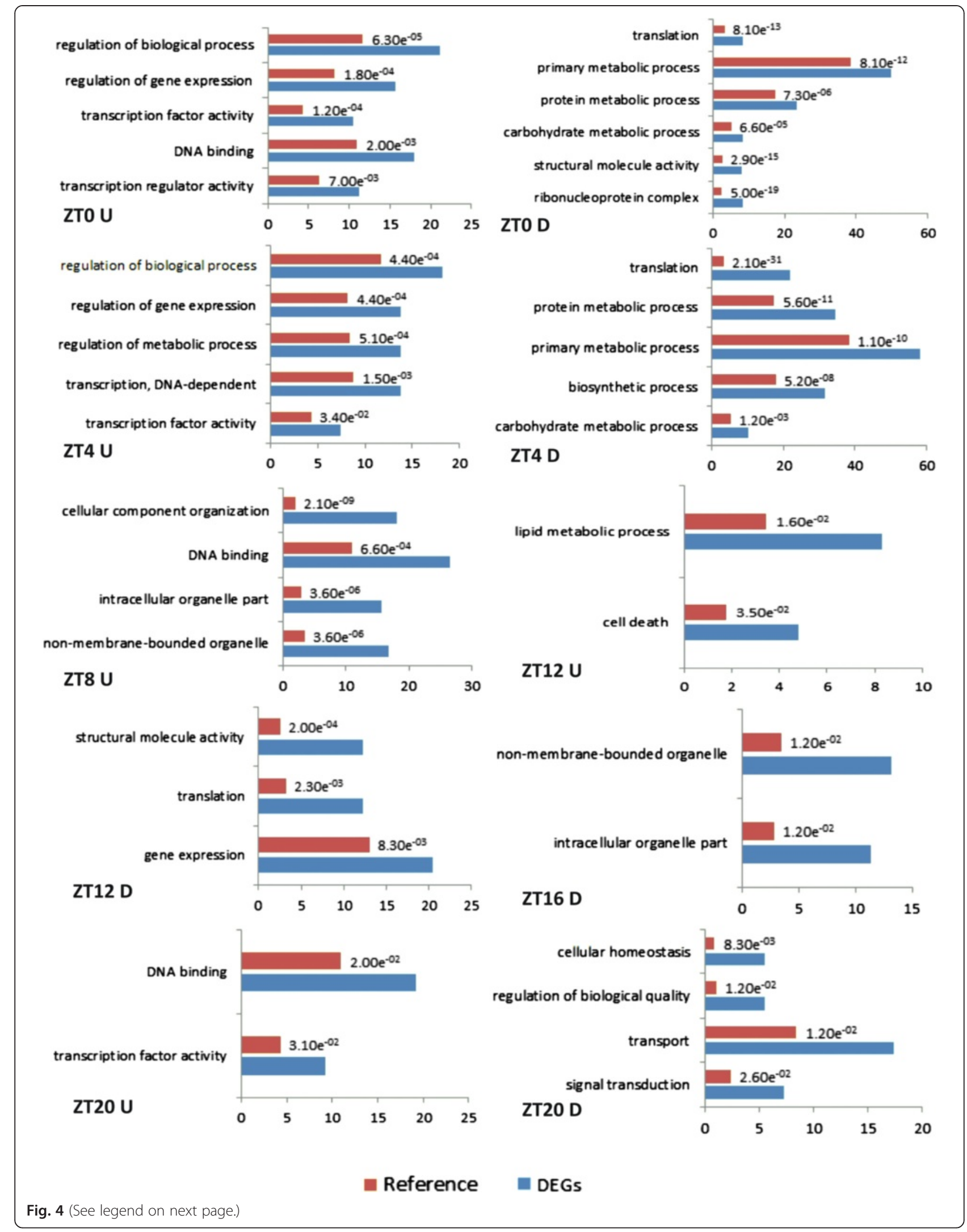


(See figure on previous page.)

Fig. 4 Enrichment analyses of functional roles. Genes were associated with Gene Ontology terms (Biological Process, Molecular Function and Cellular Component) and compared to the soybean genome (False Discovery Rate [FDR], $p$-value < 0.05) using AgriGO and REVIGO. Main enriched processes are presented for each time period. Red bars (Reference): genes present in soybean genome; Blue bars (DEGs): differentially expressed genes

PR-proteins $(n=84)$, and light $(n=77)$, among others $(n=92)$ (Fig. 5b, Additional file 6).

To assess the association levels of the expression datasets (RPKM) between time periods, a matrix of similarity was calculated using the Pearson correlation coefficient (Fig. 6). Positive correlations were observed for all comparisons in the control and water deficit sets of genes at the $0.00 p$-value. In the control plants, major differences were identified in the groups ZT0 - ZT8 $(r=0.75)$, ZT4 ZT16 $(r=0.76)$, ZT8 - ZT16 $(r=0.66)$, and ZT8 - ZT20 $(r=0.64)$. The main dissimilarities between the water deficit treated groups occurred in the ZT0 - ZT16 $(r=0.66)$, $\mathrm{ZT} 4-\mathrm{ZT} 16(\mathrm{r}=0.53)$, ZT8 - ZT16 $(\mathrm{r}=0.52)$, and ZT8 ZT20 $(r=0.48)$ time periods. Although expression for these sets of genes varied in plants under normal water availability, these groups decreased similarity under stress, indicating that the applied water deficit influenced the expression of those genes. Additionally, in the stressed group, the ZT0-ZT8 $(r=0.72)$, ZT0-ZT12 $(r=0.72)$, ZT4-ZT12 $(r=0.76), \quad Z T 4-Z T 20 \quad(r=0.61)$, and ZT12 - ZT20 $(r=0.74)$ comparisons showed significant changes in the associations between different time periods (Fig. 6).

Considering the 14,484 genes shared between the control and stress conditions, 372 were specific for the stress response (2.56\%), and, of those, 198 were responsive to abiotic stress, primarily heat and drought/salt stresses (Additional file 7). This group of genes was distinct from those exclusively observed in the control or stress treatments. Although many genes specifically expressed in response to stress were detected in both control and stressed plants, it is important to emphasize that these results represent gene expression data derived from RPKM values. Since such genes appeared exclusively in

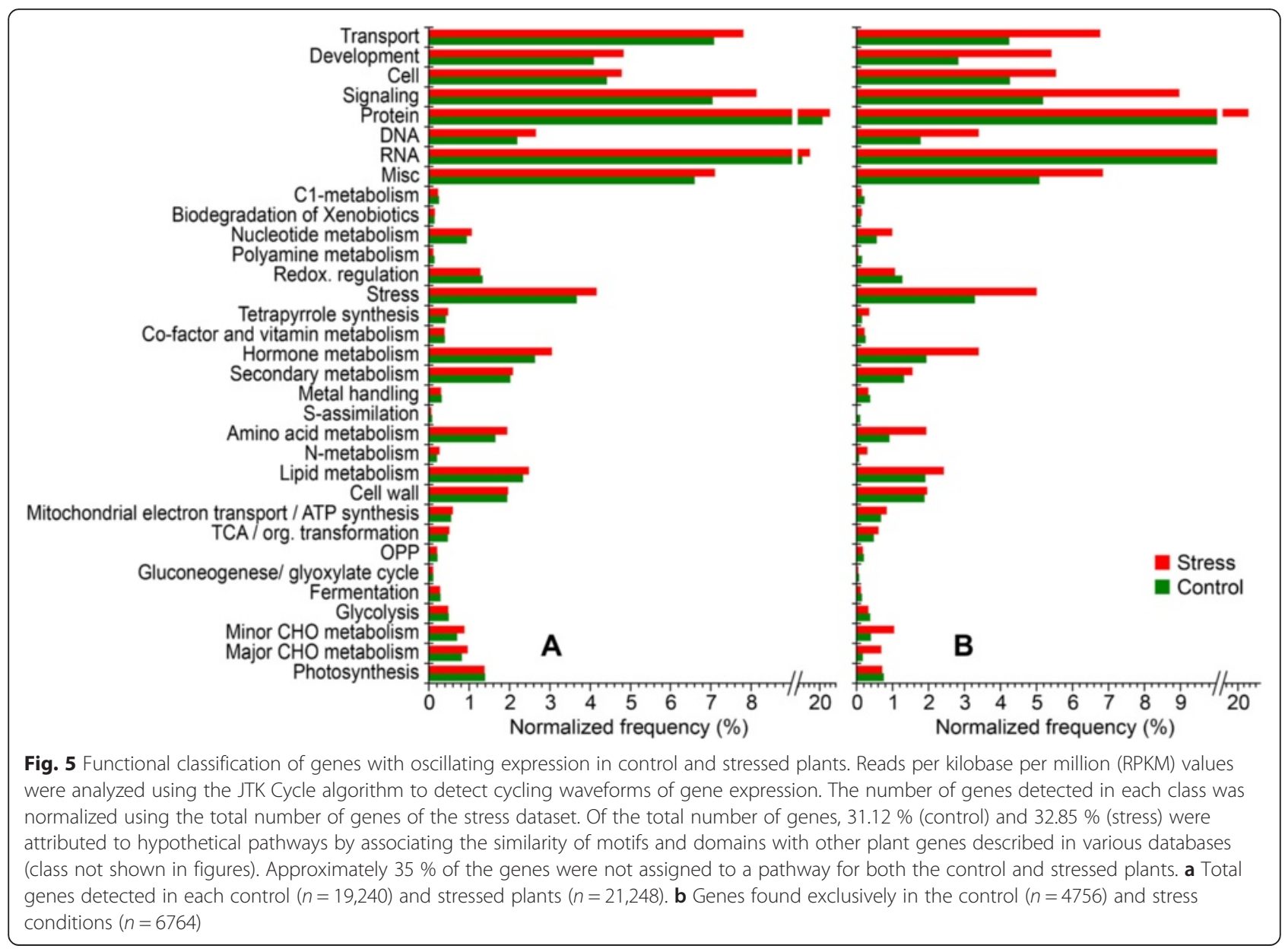




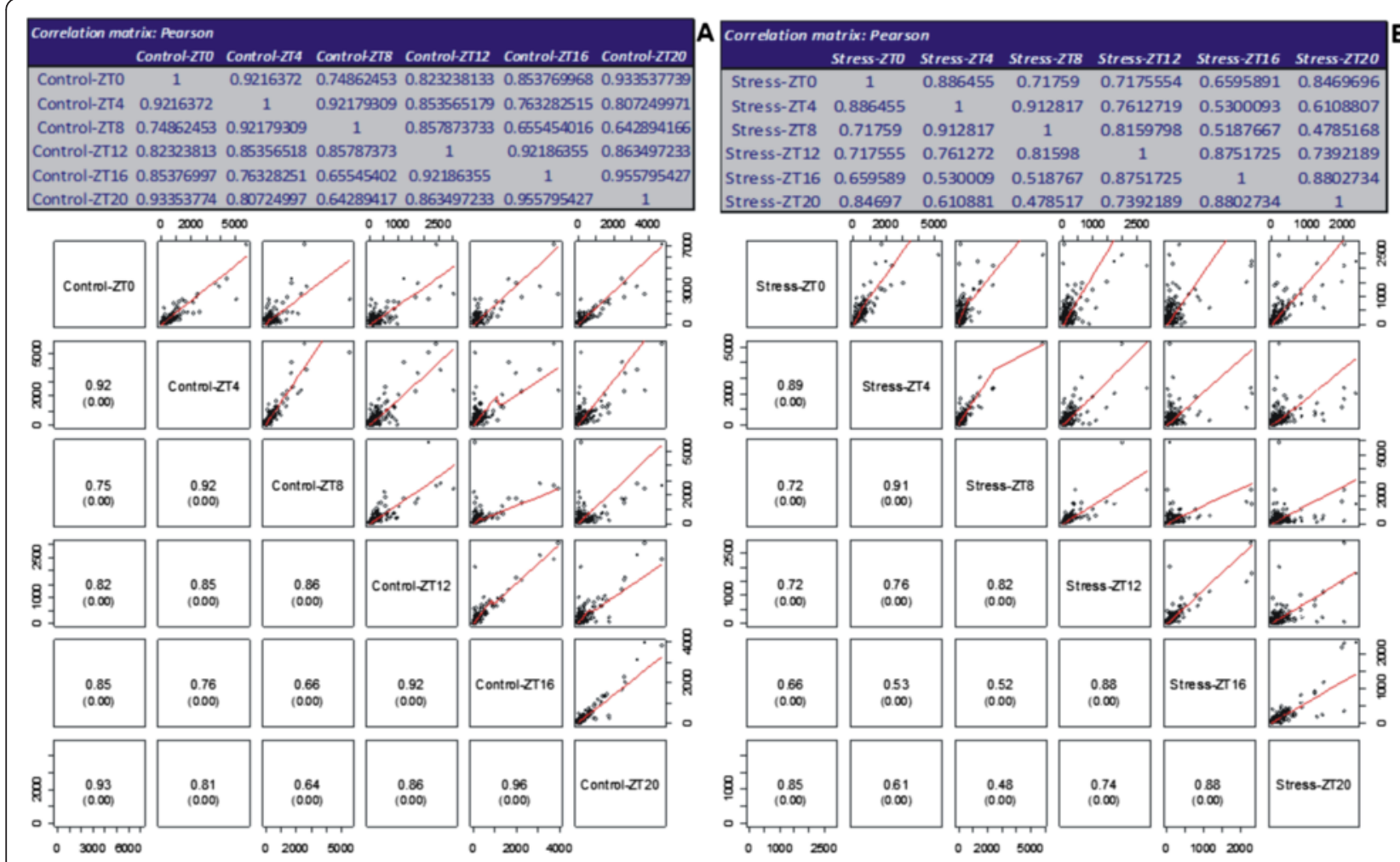

Fig. 6 Pearson's correlation matrix. Gene expression data (RPKM) from the (a) control and (b) stress groups were individually analyzed through pairwise comparisons to assess the similarities and dissimilarities among time periods. The matrices of scatterplots indicate the association, correlation, and $p$-value (in parentheses) of each comparison

control or stressed plants, the ratio of differential gene expression could not be analyzed using the software package edgeR; thus, it was not possible to infer about their up- or down-regulation under water deficit condition. According to the rhythmicity analysis of the 14,484 common genes, $12.64 \%$ of the genes under water deficit shortened their predicted period of expression (PER) from 24 to $20 \mathrm{~h}(n=1247)$ or from 24 to $22 \mathrm{~h}(n=584)$ (Additional file 7). Similarly, $56.80 \%$ of the common genes also shifted their phase (predicted phase) under water deficit. Most $(n=5995)$ advanced their expression phase under stress condition, modifying their peak from 20 to $22 \mathrm{~h}$ (in control) to $0 \mathrm{~h}$ (water deficit condition) (Additional file 7). Conversely, a set of 2233 genes delayed the LAG phase, showing their expression peak at $20-22 \mathrm{~h}$ in stress conditions, rather than at $0 \mathrm{~h}$ (control condition) (Additional file 7). Under water deficit, 6820 genes $(47.08 \%)$ reduced their expression amplitude (AMP), whereas 7663 (52.90 \%) increased it (Additional file 7).

Some genes that showed oscillating expression were plotted on graphs to observe such patterns during the day (Fig. 7, Additional file 8). Glyma07g04310 (coding for germin-like protein 1) and Glyma16g00980 (germin 3) were not expressed in response to water deficit (both were down-regulated in ZT0-ZT4; Additional file 8), but their expression was shaped by time of day in control and stressed plants (Fig. 7a and b, respectively). Similarly, Glyma13g16960 (germin-like protein 1) also exhibited significant oscillations in both control and stressed plants, although it was induced during ZT8 (Fig. 7c). For all three genes, the PER was maintained in stressed plants, although the phase advanced and AMP was shifted, in general (Additional file 8). Oscillation patterns for genes differentially expressed during the light periods were observed for Glyma06g08540 (BURP domain-containing protein), which exhibited notable increased expression from ZT0 (4.77 fc) to ZT4 (10.08 fc) (Fig. 7d), and for Glyma12g34570 (BURP domaincontaining protein), whose oscillation was observed specifically in control plants (Fig. 7e). Glyma04g08410 (BURP domain-containing protein) showed differential expression at midday $(2.93 \mathrm{fc})$, in ZT12 (2.32 fc) and in the dark period at ZT20 (2.34 fc) (Fig. 7f, Additional file 8). Interestingly, the expression peaks observed in these time periods were identified as differential in response to water deficit. However, overall, each control and stressed plant showed significant contrasting expression profiles (Fig. 7f). Glyma12g34550 (BURP domain-containing protein) was up-regulated during 


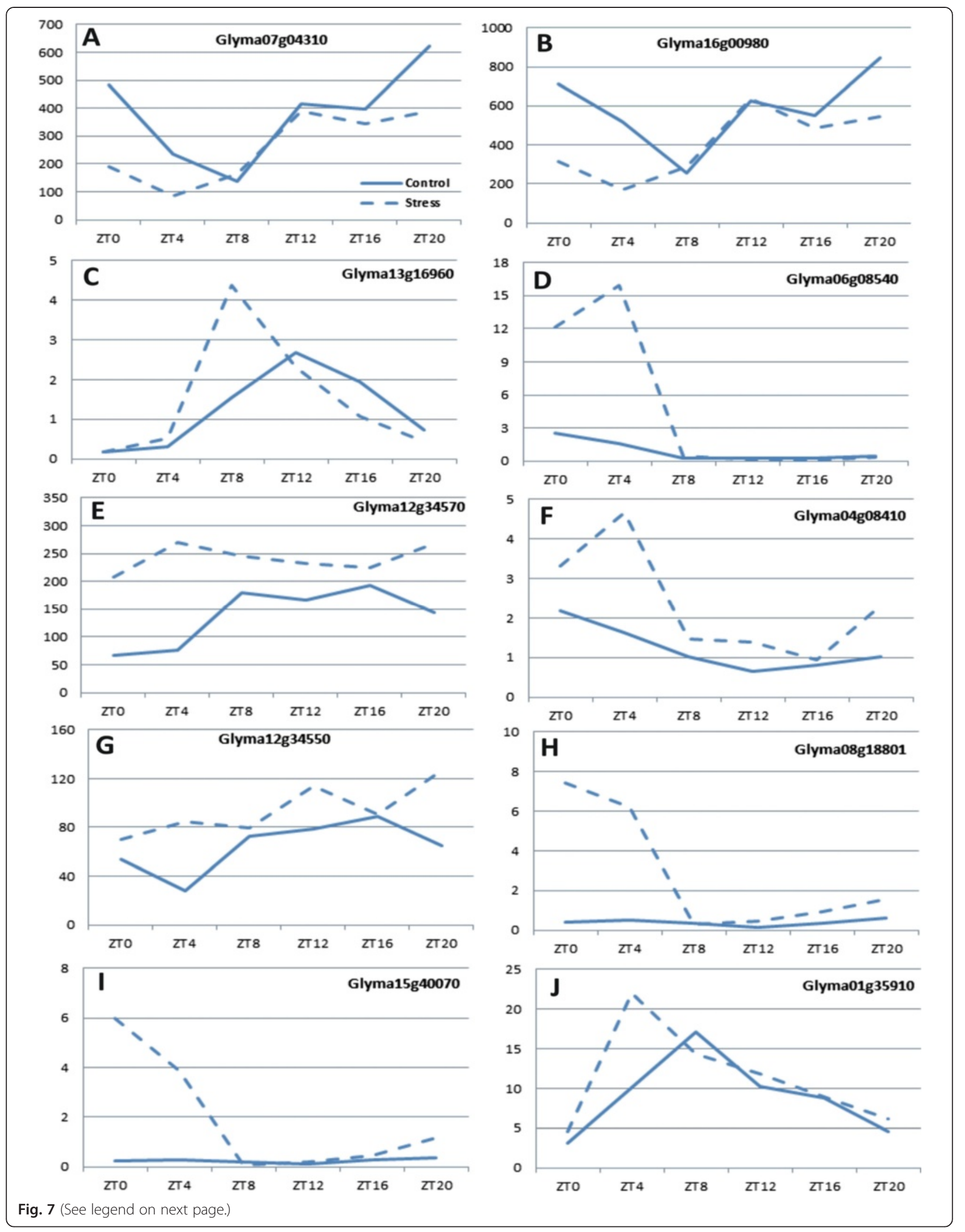


(See figure on previous page.)

Fig. 7 Oscillations of expression of genes observed in soybean response to water deficit stress. Waveforms were detected through rhythmicity analysis using the JTK Cycle algorithm. a Glyma07g04310, coding for germin-like protein 1; b Glyma16g00980, germin 3; c Glyma13g16960, germin-like protein 1; d Glyma06g08540, BURP domain-containing protein; e Glyma12g34570, BURP domain-containing protein; f Glyma04g08410, BURP domain-containing protein; g Glyma12g34550, BURP domain-containing protein; h Glyma08g 18801, 9-cis-epoxycarotenoid dioxygenase 5; i Glyma15g40070, 9-cis-epoxycarotenoid dioxygenase 3; j Glyma01g35910, 9-cis-epoxycarotenoid dioxygenase 4

midday ( $3.14 \mathrm{fc})$ and pre-dawn periods ( $2 \mathrm{fc})$, however, the diel fluctuations of expression were significant only in control plants (Fig. 7g, Additional file 8). According to the rhythmicity analysis, Glyma08g18801 (9-cisepoxycarotenoid dioxygenase 5) and Glyma15g40070 (9-cis-epoxycarotenoid dioxygenase 3) showed similar expression profiles, peaking in ZT0 and ZT4 exclusively in stress condition (Fig. $7 \mathrm{~h}$ and i, respectively) with ratios from 10 to 25 times higher (Additional file 8). Interestingly, Glyma01g35910 (9-cis-epoxycarotenoid dioxygenase 4) showed a different profile under water deficit condition, with peak expression in ZT4. However, in control condition peak expression was observed in ZT8, levels that resulted in a ratio of $2.24 \mathrm{fc}$ (Fig. 7j). The PER $(20 \mathrm{~h})$ and phase $(10 \mathrm{~h})$ of this oscillating expression were maintained after plants became droughtstressed, but AMP was increased from approximately 2.316 (control) to 3.639 (stress condition) (Additional file 8).

In accordance with our goals, we compared the set of genes that were differentially expressed (DEGs) in response to water deficit (Fig. 1, Additional file 1) to genes with putative cycling expression in either control or drought-stressed plants (Fig. 5, Additional files 4 and 5). Among the 4866 DEGs in response to water deficit, 3791 (3040 unique genes) were observed with oscillating expression in at least one analyzed condition. In ZT0, a large set of differentially expressed genes was composed of more down- $(n=1353)$ than up-regulated genes $(n=478)$ (Additional file 8). In ZT4, we observed the opposite expression pattern, with 674 up- and 374 downregulated genes (Additional file 8). During the ZT8 (126 up- and 21 down-regulated) and ZT12 (248 up- and 39 down-), oscillations in gene expression occurred predominantly for up-regulated genes (Additional file 8). In the midnight period (ZT16), the pattern was similar to the pattern from early morning (ZT20), showing more down- $(n=79)$ than up-regulated genes $(n=39)$ (Additional file 8). Conversely, there was a balanced set of genes in the pre-dawn (ZT20) period, with 193 up- and 167 down-regulated genes (Additional file 8). Regarding the ZT0 - ZT8 periods, 250 genes overlapped within ZT0-ZT4. However, approximately $96.6 \%$ of the genes from ZT8 were uniquely expressed. Few genes exhibited overlapped expression within ZT12-ZT20 $(n=8)$ and ZT16-ZT20 $(n=10)$. No genes were found between ZT8 and ZT12. Of the 138 expressed genes identified in common to both the light and dark periods, 78 were expressed in the early morning (ZT0) and also detected at the pre-daw period (ZT20).

All DEGs that exhibited oscillating expression (Additional file 8) were mapped to the main pathways involved in plant response to stress (Fig. 8, Additional file 9). Genes expressed in ZT0 were included in many pathways, including signaling and cell wall metabolism, that were repressed in response to water deficit during this time period. Conversely, some genes from the abiotic stress class were highly induced in a similar manner to hormone (ABA) signaling pathways and heat-shock proteins (HSPs) (Fig. 8, Additional file 9). The jasmonate pathway was also induced, as indicated by the expression of three genes related to lipoxygenase (Glyma07g00920), allene oxide synthase (Glyma07g21100), and 12oxo-PDA-reductase (Glyma14g39790). The jasmonate pathway remained induced in ZT4 but was represented by two other genes encoding allene oxide synthase (Glyma11g13070) and 12-oxo-PDA-reductase (Glyma11g00980). Glyma07g00920, which was moderately expressed in the early morning (ZT0), also was highly up-regulated during pre-dawn (ZT20) (Fig. 8, Additional file 9). According to these results, some genes detected in a specific pathway were not expressed during the entire diel period, but genes encoding different proteins seem to be involved in the same stress-related pathways. The expression of genes related to PR-proteins is another example of how different genes are involved in maintaining increased or decreased metabolic activity, since most genes were distinct in each time period, and only one down-regulated gene at dawn (ZT0) (Glyma20g33740) was positively regulated in ZT12 (transition light-dark). All other genes related to PR-proteins in ZT12 were exclusively detected at this time period (Fig. 8, Additional file 9). In the abiotic stress class (heat, cold, drought, salt, and wounding), genes were predominantly up-regulated (expression ranged from 6.05 to $14.05 \mathrm{fc}$ ). Similar profiles were observed for redox-state metabolism; HSPs; ABA and other genes involved in hormone signaling; the transcription factors MYB, WRKY, DOF, and ERF; and for the class of secondary metabolites. Because the sets of DEGs were smaller in the ZT8 and ZT16 time periods, the profiles observed for the metabolic pathways were under-represented. In ZT8, few genes related to signaling and hormone signaling (ABA and jasmonate) were up-regulated. Conversely, such signaling and hormone 


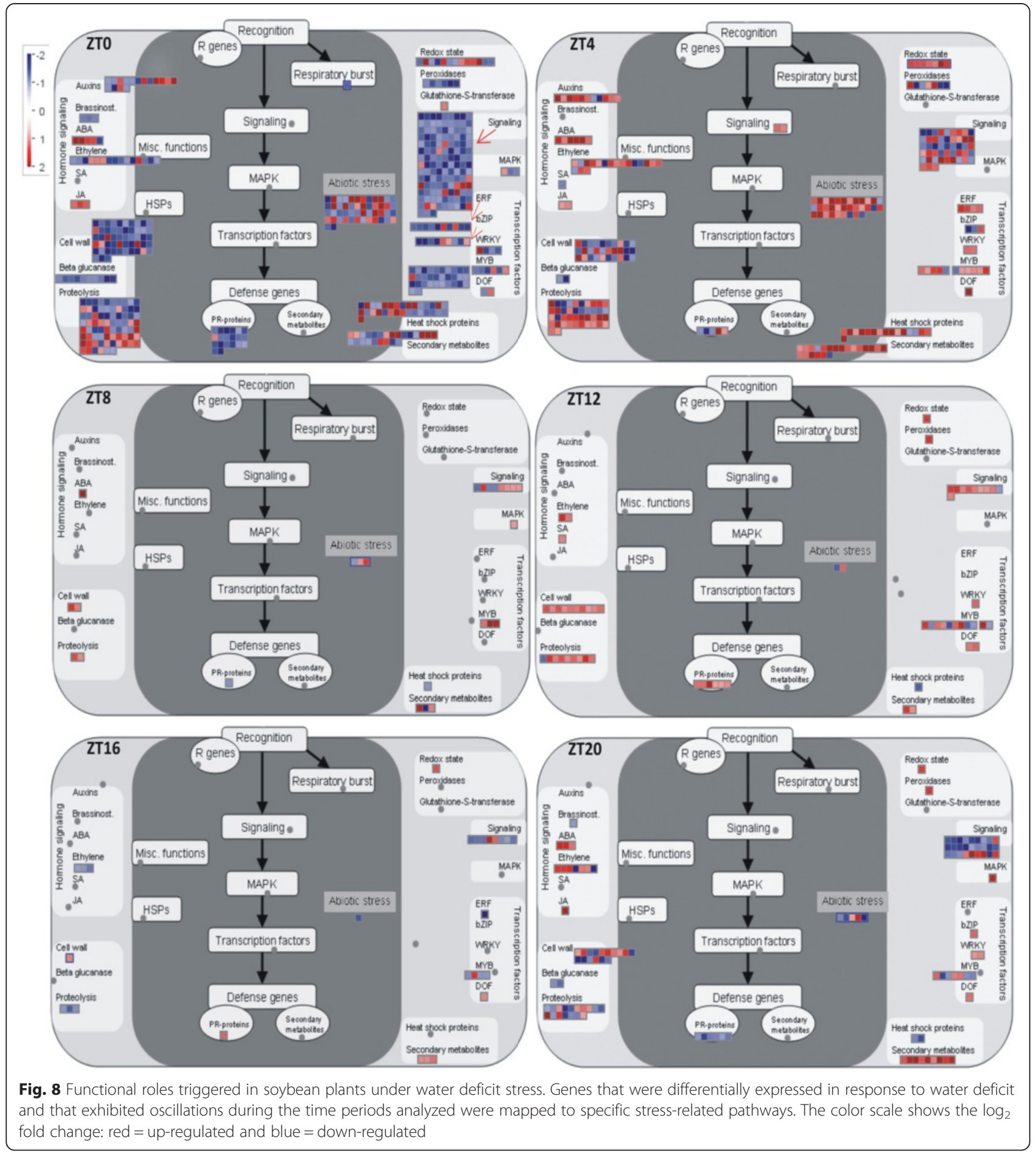

signaling (ethylene) processes became repressed in ZT16. Although many genes were down-regulated during pre-dawn (ZT20), up-regulated ones were identified as involved in the processes of secondary metabolism, transcription factors, $\mathrm{ABA}$ and ethylene hormone signaling, and redox-state metabolism (Fig. 8, Additional file 9).

\section{Validation of gene expression}

Expression of genes related to proteins involved in plants' responses to water deficit stress as the Remorin (Glyma19g32280), Gols (Glyma19g40680), DREB1 (Glyma14g09320), RAB18 (Glyma09g31740) and bZIP (Glyma02g14880) were analyzed by the method $2^{\wedge-(\Delta C t)}$. A ratio of expression (fold-change) was calculated by 
dividing the expression detected in drought-stressed plants by the one observed in control (Fig. 9, Additional file 10). The gene-coding for RAB18 presented the highest fold-change in both ZT0 and ZT4 time-periods (Fig. 9, Additional file 10). In general, linear equation demonstrated a good correlation between both experiments. Genes used in this analysis represent a subset from those evaluated in Marcolino's et al. [20] study.

\section{Discussion}

The biological processes of plants are coordinated with physical and biochemical reactions, such as water intake, gene expression control, protein synthesis, and posttranslational modification. Receptors in cell membranes communicate adverse signals from the surrounding environment to synchronize metabolic processes [7, 24, 25]. To protect themselves, plants under water deficit change their normal cellular activities, such as movement, secretion, enzyme activity, and gene expression. In Arabidopsis, transcriptome reconfiguration in response to drought is associated with distinct hormonal and stress response pathways induced at different times of the day [17]. Soybean genes modulating responses to water deficit stress were expressed in different time periods during the course of $24 \mathrm{~h}$ (Figs. 2 and 3, Additional file 2). Whereas many genes involved in translation and bioenergetic processes were repressed in most time periods, enriched processes, such as gene expression and transcription factor activity/ DNA binding, were predominantly up-regulated until late day (Fig. 4) and were detected again during the pre-dawn period. Most of these transcription factors (GATA, heat shock, ERF domain, AP2, ABFs, and bZIP proteins) are directly involved in plant responses to water deficit.

Studies have demonstrated the circadian rhythm control of gene expression for different plant species [4, 5, 14-16]. In Arabidopsis, the number of genes under circadian regulation has been estimated to be hundreds $[8,9]$ to thousands regulating the control of auxin signaling [11]. Covington et al. [7] estimated that one-third of the expressed Arabidopsis genes are circadian clockcontrolled. In maize, $10 \%$ of the 13,000 analyzed transcripts showed circadian pattern expression [5]. Our results suggest that $35 \%$ of the soybean genome (Glyma 1.1) showed oscillating expression in a diel period in plants growing under normal availability of water (Additional files 4 and 6). Seventy five percent of those genes also were detected in plants under water deficit, many of them shifted their PER or phase in such adverse condition (Additional file 5). Hsu and Harmer [24] demonstrated that differences in gene expression, even small, are associated with changes in phase and can influence expression. Additionally, dissimilarities observed in the set of expressed genes between some light/dark periods (ZT4 - ZT16, ZT8 - ZT16, and ZT8 ZT20; Fig. 6a) reinforce the evidence of daily fluctuations in gene expression in soybean plants growing under normal conditions. Transcriptome changes observed for Populus submitted to drought depended on the time of day at which they were measured [16]. Similarly, in Arabidopsis plants, the interaction with diurnal regulation was predominant in modulating the transcriptome responses to cold stress [15]. Taken together, these data indicate that the time of day might play an important

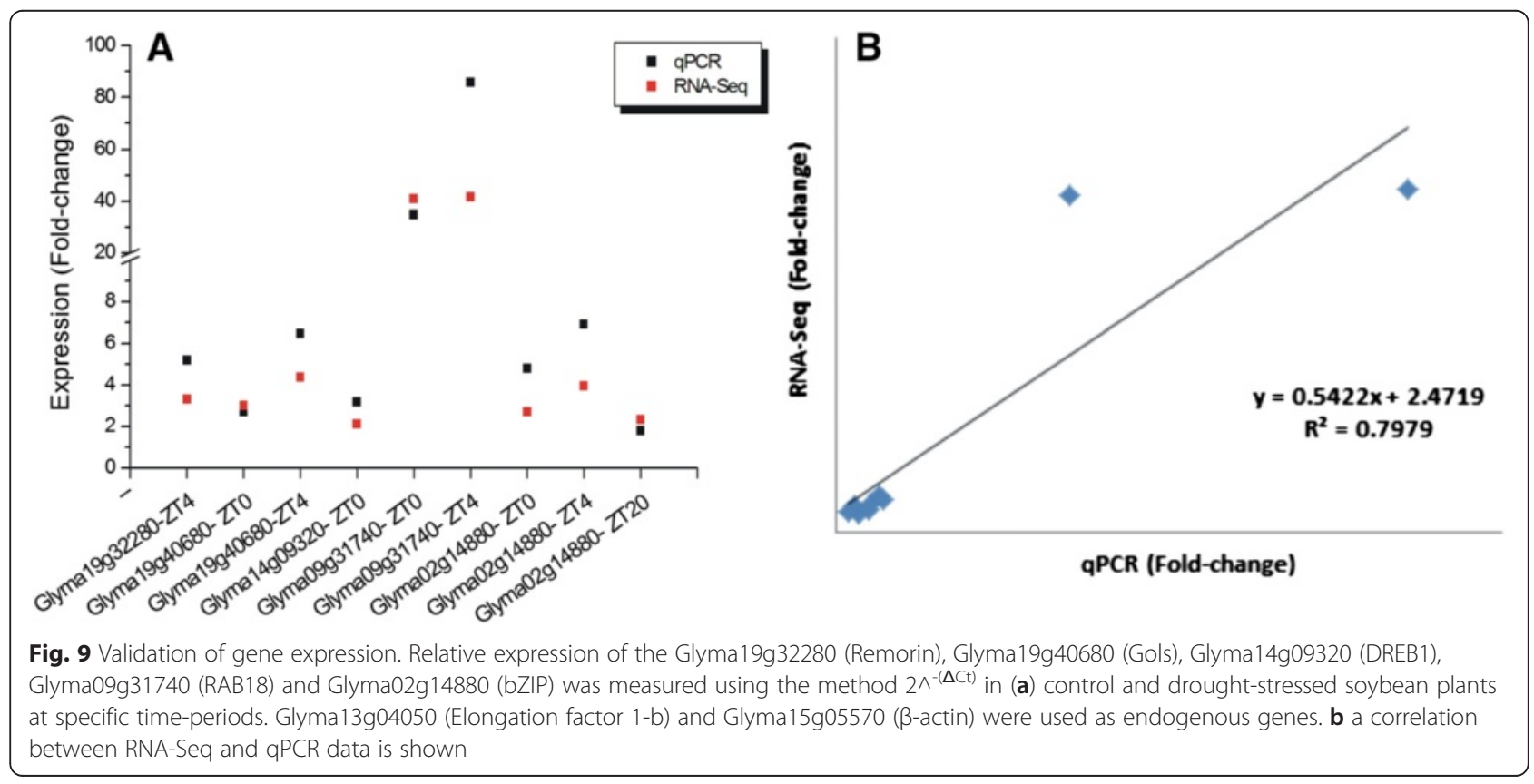


role in regulating the expression of many soybean genes in both normal and water deficit stress condition.

Gene expression changes dynamically during the course of normal development and in response to other organisms, physical damage, or adverse environmental conditions. Such behavior was observed in the expression profiles of NCED enzymes (Fig. 3), both rate-limiting components in ABA biosynthesis from the cleavage of carotenoids. In addition to the role that this phytohormone plays in normal development, ABA content is also increased in response to water deficit, preserving cell water content due to stomatal closure. In Arabidopsis, NCED5 acts in association with NCED3 to synthesize ABA under normal conditions and in response to stress conditions [26]. Genes related to ABA biosynthesis (including the NCEDs) have been linked to circadian regulation [7]. Interestingly, genes for NCED5 and NCED3 (Fig. 7h and i) showed higher expression levels compared to NCED genes. However, the two exhibited similar expression peaks at ZT0 - ZT4 under stress condition (but not in the control). The gene for NCED4 (Glyma01g35910; Fig. 7j) also was detected in the light period, but its waveforms were distinct for control (ZT8) and stress conditions (ZT4). Such changes in oscillation can be attributed to delayed amplitude under stress condition since the period and phase remained unchanged (Additional file 8). Although the diurnal fluctuation of ABA levels in tobacco plants has been implicated to occur at the end of a light period [13], as detected to the expression of NCED4 gene (Fig. 7j) in plants under normal water supply, such oscillation appeared to be limited to light under stress condition (Fig. $7 \mathrm{~h}-\mathrm{j}$ ), the period that stomatal closure is needed to avoid water loss by evapotranspiration process.

Similarly, the gene coding for the oxidative stress 3 (OXS3) protein (Fig. 3) exhibited up-regulation in response to light (ZT0) but not dark (ZT20) (Additional file 1). This OXS3 protein is related to cadmium ion tolerance, as demonstrated in mutants of AtOXS3 that were unable to enhance stress tolerance [27]. The authors have also associated the AtOXS3 gene with regulation by light, suggesting a putative role for this protein in protecting the cell against photooxidation [27]. We found eight paralogs in the soybean genome that were related to the Arabidopsis OXS3 (AT5G56550.1); three of them were identified in this study as differentially expressed in response to water deficit. In addition to Glyma11g33040 reported above, Glyma01g00930 and its paralog Glyma07g15070 also were up-regulated in ZT0 (Additional file 1). Although we do not have information about the functional role of those proteins in the water deficit stress scenario, it is tempting to speculate on their importance in the soybean transcriptome since drought events can produce oxidative stress in the plants [28, 29]. Additionally, Glyma11g33040 showed a cycling pattern when expressed in normal condition (control plants) (Additional file 1), suggesting that its expression can be influenced by time of day.

Under water deficit, it was observed that genes were predominantly down-regulated at dawn (ZT0) (Fig. 1, Additional file 1). The expression levels of 192 downregulated genes in ZT0 did not increase at midday (ZT4) (Additional file 2). Some of these genes that exhibited down-regulated profiles in ZT4 are involved in basal metabolism, such as protein synthesis and DNA metabolism. In addition, the carbohydrate metabolic process (Fig. 4), down-regulated at dawn and midday, represented a significant change in plant bioenergetics metabolism by reducing the synthesis of organic compounds and the breakdown of carbohydrates. This suggests that during these time periods, energetically expensive processes are being partially arrested, and energy resources are being redirected to activate protective mechanisms. According to Fraire-Velázquez and Balderas-Hernández [30], the optimization of cellular energy resources during stress is essential for plant acclimation. Dhaubhadel et al. [31, 32] reported the accumulation of HSPs in Brassica napus seedlings under heat stress. This significant accumulation resulted from higher HSP synthesis even when the mRNA levels were lower in treated seedlings compared to controls. Such regulation mechanisms might act under post-transcriptional control, suggesting an advantageous ability of the plant machinery to save energy or drive it to maintain the translational apparatus during stress events.

In our study, the genes encoding germin and germinlike proteins are examples of genes that were repressed in early morning until midday (ZT0 - ZT4). Germin has been associated with many processes important for plant development and defense [33-35]. In the soybean genome, 36 genes annotated as germin or germin-like proteins are associated with the cupin superfamily, which includes a variety of enzymes and non-enzymatic seedstorage proteins. We detected the paralogous Glyma07g04310 and Glyma16g00980 down-regulated in both ZT0 and ZT4, which are associated, by suggestive evidence, with nutrient-reservoir activity. Conversely, Glyma13g16960 (ZT8) and Glyma01g04450 (ZT16) were up-regulated under water deficit $(2.82 \mathrm{fc}$ and $2.27 \mathrm{fc}$, respectively) (Additional file 1). Germin-like protein genes (Fig. $7 \mathrm{a}-\mathrm{c}$ ) exhibited similar oscillation profiles with respect to control plants since they all showed expression peaks in ZT12. In general, under stress, genes advanced phases and shifted amplitude. The results obtained for Glyma13g16960 (Fig. 7c, Additional file 1) indicate that this gene is involved in the response to water deficit stress but also indicate that different germin-like protein genes expressed in soybean growth and development might oscillate levels with the time of 
day. The diverse expression patterns of germin-like protein genes during soybean development also were reported by Lu et al. [36]. Their study demonstrated that these genes are involved in enhancement of salt tolerance, and they exhibit expression fluctuations in darkness, suggesting a circadian clock feature [36]. In Arabidopsis, the ortholog (AT1G72610.1 [germin-like protein 1]) of Glyma16g00980 as well as Glyma07g04310, as other germinlike proteins (GLP2 and GLP3), are associated with the extracellular matrix, hypothetically acting in developmental processes or stress responses [37]. Additionally, the circadian regulation for Atger3, a germin-like cell wall protein from Arabidopsis, seems to occur at the beginning of the night [38]. In Sinapis alba, a long-day plant from the same family of Arabidopsis, circadian oscillation was associated with a transcript that encodes a germin-like protein that exhibits a transcription peak during dark periods (ZT12-ZT16) [39]. Likewise, circadian regulation also was suggested for the germin-like protein 9 (GLP9) in Arabidopsis [40].

Conversely, genes that were positively regulated in response to water deficit, such as the BURP domaincontaining protein (Fig. $7 \mathrm{~d}-\mathrm{g}$ ), were detected in various time periods (ZT0, ZT4, ZT12, and ZT20). This class of protein contains a conserved domain found in diverse plants and is putatively involved in the localization of proteins within the cell wall matrix through association with a structural domain that might target sites for intermolecular interaction [41]. Although the function of many BURP proteins is unknown, specific elements have been characterized, and the functional role of these proteins is associated with normal plant metabolic processes, such as seed development [42]. Moreover, the genes encoding the BURP domain-containing proteins also are involved in rice responses to abiotic stresses [43]. Some members of the rice BURP family are responsive to cold, ABA, and drought and salt stresses and can be induced by a single stress condition or combination of treatments. This family also exhibits temporal and spatial expression pattern differences [43]. In soybean plants, the BURP family contains 23 genes that have been classified into five subfamilies (BNM2, USP, RD22, PG1 $\beta$, and BURP V). Although these genes possess no tissue specificity, they are expressed in response to stress. In particular, genes from the RD22 subfamily are the most responsive to ABA, PEG treatments, and salt stress [44]. For the GmRD22 protein (Glyma06g085400), the BURP domain seems to have an important role for determining its apoplast localization [45]. Furthermore, this protein-coding gene (GmRD22 [Glyma06g085400]) exhibits potential responses to salt and osmotic stresses [45]. In this study, we identified four up-regulated BURP genes from the classes previously determined by $\mathrm{Xu}$ et al. [44]: RD22 (Glyma06g08540 and Glyma04g08410), USP (Glyma12g34570), and
Glyma12g34550 (a gene not included in the classification). Expression levels exhibited by these genes indicate the responsiveness of BURP-domain-containing proteins to water deficit, and the oscillation patterns detected in plants under normal development show the regulation of gene expression also might be influenced by time of the day.

The DEGs that showed oscillating expression in control or drought-stressed conditions (Additional file 8) played functional roles as regulatory genes in hormone signaling, cell communication, and abiotic stress-related pathways (Fig. 8, Additional file 9). Few genes involved in biotic stress responses (signaling and PR-proteins) also were down- or up-regulated during this time. Genes responsive to multiple stresses are often detected in plants under adverse conditions, since a common set of biological processes triggered by genes induced in both events converge on similar downstream responses [46, 47]. Our results showed that several hormone signaling genes, including genes related to jasmonate hormone metabolism, were observed in the early and late morning (ZT0 and ZT4), and redox reactions were up-regulated in most of the time periods (Additional file 9). Increased hormone signaling and fluctuations in the cellular redox status have been associated with plant responses to stress, and, in addition, circadian regulation also has been implicated in these processes [48]. With respect to the classification of functional roles assigned to DEGs, a similar fraction of genes down- and up-regulated in the same class can be observed. For instance, Glyma07g04310 (Fig. 7a) and Glyma16g00980 (Fig. 7c), both coding for the same type of protein, showed distinct expression patterns at different time periods. Evaluation of gene expression performed at a single time point during the day can only provide information about the plant's responsiveness to drought during that specific period in which plants were sampled. In this context, soybean gene networks induced in response to stress and modulated over a diel period appear to be a significant feature in the acclimation process, and exploring these interactions might provide novel insight into how plants respond to water deficit.

\section{Conclusion}

Changes in the gene expression profile of soybean leaves triggered in response to water deficit stress were dynamically modulated in the diel period. Such results demonstrated the importance of analyzing different time periods to characterize plant responses to stress. Analysis of rhythmicity indicates that many putative cycling genes are expressed in soybean leaves under normal development. When plants became stressed, a large number of the cycling genes found in the control plants showed a different rhythmic pattern. In addition, other genes showed fluctuations under stress conditions. Genes that were differentially 
expressed in more than one time period and whose expression oscillated during the course of the day provide evidence suggesting that time of day contributes to regulation of stress responses in soybean.

\section{Methods}

\section{Plant growth and water deficit treatment}

Soybean plants from cultivar BR16 were cultivated until the V1 developmental stage [49] in a growth chamber under specific conditions. Seeds were germinated in SuperSoil $^{\circ}$ (Scotts Miracle-Gro Company, Marysville, Ohio) at a temperature of $28 / 20^{\circ} \mathrm{C}$ (day/night, respectively), relative humidity of $80 \%$, and photoperiod of $14 \mathrm{~h}$ day (under $500 \mu \mathrm{mol} \mathrm{m}{ }^{-2} \mathrm{~s}^{-1}$ of white light) $/ 10 \mathrm{~h}$ night. The experimental design was completely randomized and included two treatments (control and water deficit), six time points, and six biological replicates for each treatment/time point. Soybean field capacity was determined using the gravimetric humidity (GH) method to establish the percentage of water in the soil [50]. We previously estimated the water volume needed to reach a $100 \%$ soil field capacity by weighting the pots daily to calculate the ratio between its fresh and dry weight. We also performed same analysis in plants after withholding irrigation to evaluate the decrease of the soil field capacity under such chamber growth conditions.

We established a $70 \%$ soil field capacity to grow all plants during 14 days. At the $15^{\text {th }}$ day irrigation was suspended to initiate the water deficit treatment: control plants were maintained at $70 \%$ and the stressed-plants were monitored periodically until soil field capacity reach $30 \%$, approximately (3 days after starting the stress treatment). At this condition, leaves were sampled in six timepoints with consecutive 4-h intervals. Plants' harvesting initiated at 8:00 $\mathrm{h}$ am (ZT0/dawn) and followed as: $12 \mathrm{~h}$ am/ZT4, midday; $16 \mathrm{~h} / \mathrm{ZT} 8$, late day; $20 \mathrm{~h} / \mathrm{ZT} 12$, transition light-dark; $24 \mathrm{~h} / \mathrm{ZT} 16$, midnight; and $4 \mathrm{~h} /$ ZT20, pre-dawn. By convention, Zeitgeber time (ZT times) was used to indicate when light period started (ZT0) and to associate the six time-points to the respective time of day. Leaves were collected, immediately frozen in liquid nitrogen and stored at $-80^{\circ} \mathrm{C}$.

\section{Library construction and sequencing run}

Total RNA was extracted from leaves using the RNA Plant Reagent ${ }^{\circ}$ according to the manufacturer's instructions (Ambion, Austin, TX) and treated with DNAse (Invitrogen, Carlsbad, CA). Following analysis of RNA quality and integrity in a Bioanalyzer (Agilent, Palo Alto, CA) (only samples with a $\mathrm{RIN} \geq 8.0$ were used), equimolar quantities of purified total RNA samples from each of two biological replicates were pooled into one template for library synthesis. For each time period/treatment were synthesized three independent libraries. The Ovation RNA-Seq (NuGEN Technologies, San Carlos, CA) method was used to enrich cDNA libraries for coding and regulatory sequences [51]. Moreover, this method was suitable for use with the Illumina platform since it permits the application of barcodes to the libraries for a multiplex sequencing strategy. Briefly, approximately $150 \mathrm{ng}$ of total RNA was mixed with the selected primers to synthesize the first strand of the cDNA using a reverse transcription polymerase. The second strand was synthesized using a single primer for reverse transcription and incorporation of analog nucleotides, followed by RNA strand degradation. Double-stranded DNA was fragmented by sonication to a median size of $200 \mathrm{bp}$ and purified with the Agencourt RNAClean XP system (Beckman Coulter, Brea, CA) using magnetic beads. Fragment ends were repaired to produce blunt ends and ligated to a pair of double-stranded fragments containing nucleotide analog-tagged adaptors. Strand selection eliminated sequences with the nucleotide analog (insert or insert + adaptor), thereby creating a sequence library with a single insert orientation. Sequences containing both adaptors were amplified using forward and reverse primers for 15 cycles, resulting in a specificstrand, rRNA-depleted cDNA library. Following qualitative and quantitative analysis using a Bioanalyzer, libraries were used to produce clusters for a $2 \times 50$ bp paired end-sequencing run. The 36 libraries were distributed into 5 lanes on a flow cell for sequencing in a Hi-Seq 2000 (Illumina). The raw data were uploaded to the GeneSifter database (Geospiza, Seattle, WA) for alignment with a reference genome.

\section{Mapping of reads and transcripts analysis}

Base calling of the raw data was performed with parsing reads according to the respective barcodes and trimmed to remove adaptors and primer sequencing. Output sequences were aligned with the soybean genome [22] (Glyma v1.1 from the Soybase database [http://www.soy base.org]) using the BWA method [52]. For an additional alignment, a post-processing toolset (Picard; http:// broadinstitute.github.io/picard/) was then used to perform local realignment, duplicate marking, and score recalibration to generate a final aligned set of genomic reads. As mapping included both unique and multiple reads, we counted these two types differentially. Unique reads were counted as a whole count, whereas multiple reads were counted proportionally for each location they were mapped to (up to five sites) when none of the adjacent unique reads appeared. Multiple reads mapped next to a set of unique reads mapped in one location were counted fully to that site. From this alignment, sequences were mapped on exons, introns, and intergenic regions, which were characterized as 
sequences outside of any annotated gene. The remaining unmapped reads were then aligned to a spliced reference created using all possible combinations of known exons to generate putative splice junction sites based on the annotation described above. These aligned data were then used to calculate gene expression by taking the total exon and known splice reads for each annotated gene to generate a count value per gene. For each library, a normalized expression value was then calculated for each gene using (1) the Reads per Mapped Million (RPM), which was calculated by taking the count value and dividing it by the number of millions of mapped reads and (2) the Reads Per Kilobase per Million (RPKM), which was calculated by taking the RPM value and dividing it by the kilobase length of the longest transcript for each gene.

\section{Differential gene expression}

For each time point (ZT times), we applied a pairwise comparison between the control and water deficit treatment using all three libraries synthesized from plants of the same time period/treatment. In the pairwise analysis, we only used genes with more than 20 mapped reads to compare gene expression using the edgeR statistical test [53]. A ratio of expression (fold-change) was performed by dividing values of gene expression under water deficit and control conditions. We combined the statistical test with the multiple-hypothesis-testing correction method of Benjamini and Hochberg [54], which calculates the False Discovery Rate (FDR), to qualify statistically significant, differentially expressed genes by avoiding inflation of type-1 errors. Differential gene expression was considered significant at an adjusted $p$-value $\leq 0.01$, and down- and up-regulation was established in the range of $\leq-2$ to $\geq 2$ fold-change (fc), respectively.

\section{Functional classification}

Differentially expressed genes were functionally classified using gene ontology (GO) terms (http://www.geneonto logy.org) from Biological Process level 3. Functional classes were normalized by dividing the number of genes in each class by the total number of genes in each set (time period). The GO terms associated with the genes also were compared with the soybean genome (V1.1) using the AgriGO tool [55] to detect which GO terms were significantly enriched or depleted in a given comparison. Analysis was performed using the following parameters: Fisher's test and multiple-hypothesis-testing correction through Hochberg FDR as statistical methods; significance level of an adjusted $p$-value $<0.05$; and the Plant GO Slim database. To decrease redundancy, results provided by AgriGO were analyzed by the REVIGO (Reduce Visualize Gene Ontology) method [23] using small similarity (0.05), the Uniprot database, and SinRel as the semantic similarity measure.

\section{Rhythmicity analysis}

The RPKM values for transcripts identified in control and drought-stressed plants were calculated separately (individual datasets for a 24-h time course with three biological replicates) and subjected to the JTK Cycles algorithm [56] to detect cycling transcripts. Period lengths (PER), phase (LAG), and amplitude (AMP) were measured using default parameters. Expression data obtained from control and stressed plants were loaded into MapMan pathways, and genes were placed into functional categories and biochemical pathways. Expression data from each time period for control and stressed plants also were compared to each other using the Pearson product-moment to generate a matrix of similarity and scatterplots.

\section{Validation of gene expression}

Experimental procedures were performed as described by Marcolino-Gomes et al. [20]. Briefly, leaf tissue from plants under normal water irrigation regime and plants drought-stressed were used in total RNA extraction, DNAse treatment and in cDNA synthesis. qPCR assays were carried out with CFX Real-Time PCR Detection System (Bio-Rad, Hercules, USA) using three independent biological replicates (each one was composed by two plants) and two technical replicates. Soybean genes assayed were Glyma19g32280, Glyma19g40680, Glyma14g09320, Glyma09g31740 and Glyma02g14880, which codify to the proteins Remorin, GOLS (Galactinol Synthase 1), DREB1 (Dehydration Element Binding 1), RAB18 and bZIP, respectively. Primers used in cDNA synthesis are described

Table 1 Primers used in qPCR assays

\begin{tabular}{llll}
\hline Gene_Name & Gene_ID & Forward primer (5'-3') & Reverse primer $\left(5^{\prime}\right.$-.3') \\
\hline GmRemorin-like & Glyma19g32280 & TGGATTGCAGTAAGCAGCAC & AGCGTGACACCACTTATCACA \\
GmGOLS-like & Glyma19g40680 & ACGGGGAAGGAAGAGAACAT & TGCACTCATCAATGGCTTGT \\
GmDREB1-like & Glyma14g09320 & GATGATGATGCCTCGGAGTTG & CGGAAAAACAAGAAAAGGGATATATC \\
GmRAB18-like & Glyma09g31740 & CAACTGGTGGCACTGGTTATGG & TGGTCATGCTGACGATGTTCCT \\
GmbZIP & Glyma02g14880 & TAATGGGAATGGGAATTGGG & GTGGTGTTGGTGTGGTGTTGTG \\
\hline
\end{tabular}

Gene sequences were searched in the Phytozome database and primers were designed using the PrimerQuest tool (Integrated DNA Technologies, Coralville, IA), from the $3^{\prime}$ untranslated region with the default settings 
in Table 1. Raw data was analyzed according to the method $2^{\wedge^{-(}(\Delta \mathrm{Ct})}$ based on Livak and Schmittgen [57], applying the Glyma13g04050 (Elongation factor 1-b) and Glyma15g05570 ( $\beta$-actin) as endogenous genes $[58,59]$.

\section{Data access}

The RNA-seq data discussed in this publication have been deposited in NCBI's Gene Expression Omnibus (GEO; http://www.ncbi.nlm.nih.gov/geo/) repository and are accessible through GEO Series accession number GSE69469.

\section{Additional files}

\section{Additional file 1: Tab-delimited file showing the genes}

differentially expressed under water deficit conditions. Data were analyzed for each time period using the edgeR statistical test. Genes that were up- (Up) and down-regulated (Down) are shown along with the ratio of differential expression, description, adjusted $p$-value cutoff (Benjamin and Hochberg False Discovery Rate [FDR] at $p \leq 0.01$ ), and the identifying numbers from the Kegg and Gene Ontology (GO) databases. Data from the rhythmicity analysis are also presented. (Adj. P) adjusted $p$-value $\leq 0.05$; (PER) predicted period (h); (LAG) predicted phase (h); and (AMP) reports amplitude. Numbers in the control and stress datasets represent the expression levels (in RPKM).

Additional file 2: Tab-delimited file showing the genes exclusively expressed or overlapping in different time periods. The gene list was organized from Edwards's diagram (Fig. 2). Genes that were up- (Up) and down-regulated (Down), the ratio of differential expression, and the description are presented.

Additional file 3: Tab-delimited file showing all enriched processes observed in each time period. Gene ontology (GO) terms were compared to the soybean genome using a statistical cutoff (False Discovery Rate [FDR], $p<0.05)$. Enrichment analyses were performed using the AgriGO database and REVIGO method. All processes (Biological Processes, Molecular Function, Cellular Component) considered enriched in each time period are shown.

Additional file 4: Tab-delimited file showing the rhythmicity analysis of the transcripts expressed in control plants. Expression data were analyzed using the JTK Cycle algorithm to detect cycling transcripts. (Adj. P) adjusted $p$-value $\leq 0.05$; (PER) predicted period (h); (LAG) predicted phase (h); (AMP) amplitude. Numbers in the control and stress datasets represent the expression levels (in RPKM).

Additional file 5: Tab-delimited file showing the rhythmicity analysis of transcripts expressed in stressed plants. Expression data were analyzed using the JTK Cycle algorithm to detect cycling transcripts. (Adj. P) adjusted $p$-value $\leq$ 0.05; (PER) predicted period (h); (LAG) predicted phase (h); (AMP) amplitude. Numbers in the control and stress datasets represent the expression levels (in RPKM).

Additional file 6: Tab-delimited file showing the genes from the rhythmicity analysis that were detected in both control and stressed -plants or exclusively in each condition.

Additional file 7: Tab-delimited file showing the rhythmicity analysis of transcripts common to control and drought-stressed plants. Expression data were analyzed using the JTK Cycle algorithm to detect cycling transcripts. (Adj. P) adjusted $p$-value $\leq$ 0.05; (PER) predicted period (h); (LAG) predicted phase (h); (AMP) amplitude. Numbers in the control and stress datasets represent the expression levels (in RPKM) and the means of three biological replicates. The PER (blue area, left axis), LAG (red area, left axis), and AMP (green line, right axis) data were plotted on graphs, and genes were organized at the same positions on the $x$-axis.

Additional file 8: Tab-delimited file showing all differentially expressed genes (DEGs) that showed significant oscillating expression in control or stressed plants. Genes that were up- (Up) and down-regulated (Down) are shown along with the ratio of differential expression, description, False Discovery Rate (FDR) cutoff (Benjamin and Hochberg at $p \leq 0.01$ ), and the identifying numbers from the Kegg and Gene Ontology (GO) databases. (Adj. P) adjusted $p$-value $\leq 0.05$; (PER) predicted period (h); (LAG) predicted phase (h); and (AMP) reports amplitude. Numbers in the control and stress datasets represent the expression levels (in RPKM).

Additional file 9: Tab-delimited file showing the pathway mapping. The MapMan database was searched for stress-responsive genes that showed cycling expression oscillation to identify the biochemical pathways that either were or were not activated in response to water deficit stress in soybean plants. Bincode (numeric index of the functional categories), BinName (functional categories), Gene ID, Description and $\log _{2}$ fold-change (negative values represent down-regulated genes) are shown for genes presented in Additional file 8.

Additional file 10: Tab-delimited file showing the data of qPCR

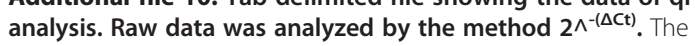
normalized data of each biological replicate is presented for control and drought-stressed plants. For RNA-Seq data, biological replicates were analyzed by edgeR statistical test and expression data is represented by a mean for control and drought-stressed plants. A ratio of expression (fold-change) was calculated by dividing of gene expression under water deficit and control conditions for each experiment.

\section{Abbreviations}

PR: Pathogenesis-Related; ABA: Abscisic Acid; DREB: Dehydration Responsive Element Binding; GO: Gene Ontology; RPKM: Reads Per Kilobase per Million; RPM: Reads Per Million; FDR: False Discovery Rate; BNM2: Microspore-derived embryo from Brassica napus; USP: Unknown Storage Protein; RD22: Responsive to Droughtness; PG1 $\beta$ : $\beta$-subunit of polygalacturonidase; BURPV: BURP domain protein from class $V$, named according to the proteins it was first identified: BNM2, USP, RD22, and PG1B; GLP: Germin-Like Protein; NCED: Nine-CisEpoxycarotenoid Dioxygenase; PEG: Polyethylene Glicol; BWA: BurrowsWheeler Alignment; ZT: Zeitgeber Time; AgriGO: GO Analysis toolkit for the agricultural community; REVIGO: Reduce Visualize Gene Ontology; PER: Period; LAG: Phase; AMP: Amplitude.

\section{Competing interests}

The authors declare that they have no competing interests.

\section{Authors' contributions}

ALN and FGH designed the experiment. ALN, JMG and TJN carried out plant growth and experimental treatment. FAR, RFP, JMG and TJN prepared the plant material; FAR, RFP and FPL also performed data analysis. FGH performed the rhythmicity analysis. ALN, HBCM and FGH contributed in reagents/materials and analysis tool. FAR and ALN wrote the paper. All authors read, revised, made contributions and approved the final manuscript.

\section{Acknowledgments}

Embrapa-Labex USA funded this project. Conselho Nacional de Desenvolvimento Científico e Tecnológico-CNPq (project \# PDE-202211/2011-8, PDE-202639/20118 , respectively) granted scholarships for FAR and RFP. Coordenação de Aperfeiçoamento de Pessoal de Nível Superior-CAPES (process \# 8075/11-5) granted scholarship for JMG. We thank the Plant Gene Expression Center USDA-ARS, Albany-CA, for the laboratory facilities. Approved for publication by the Editorial Board of Embrapa Soja as manuscript 08/2015.

\section{Author details}

'Brazilian Agricultural Research Corporation- Embrapa Soybean, Embrapa Soybean- Rod. Carlos João Strass, s/n, Londrina 86001-970PR, Brazil. ${ }^{2}$ Department of Biology, State University of Londrina, Londrina, PR, Brazil. ${ }^{3}$ Department of Crop Science, Federal University of Viçosa, Viçosa, MG, Brazil. ${ }^{4}$ Genetics and Biotechnology Laboratory, Embrapa Agroenergy (CNPAE), Brasília, DF, Brazil. ${ }^{5}$ Brazilian Agricultural Research Corporation-Embrapa Agricultural Informatics, Campinas, SP, Brazil. ${ }^{6}$ Plant Gene Expression Center, USDA-ARS, Albany, CA, USA. 'Department of Plant and Microbial Biology, UC Berkeley, Berkeley, CA, USA. ${ }^{8}$ Embrapa LABEX US Plant Biotechnology at ARS/ USDA Plant Gene Expression Center, Albany, CA, USA. 
Received: 7 January 2015 Accepted: 26 June 2015

Published online: 07 July 2015

\section{References}

1. Pedersen P, Kumudini S, Board J, Conley S. Soybean growth and development. In: Dorrance AE, Draper MA, Hershman DE, editors. Using foliar fungicides to manage soybean rust. Ohio: Ohio State University; 2005. p. 41-7

2. Xoconostle-Cázares B, Ramírez-Ortega FA, Flores-Elenes L, Ruiz-Medrano R. Drought tolerance in crop plants. Am J Plant Physiol. 2010;5:241-56.

3. Osakabe Y, Yamaguchi-Shinozaki K, Shinozaki K, Tran L-SP. Sensing the environment: key roles of membrane-localized kinases in plant perception and response to abiotic stress. J Exp Bot. 2013;64:445-58.

4. James AB, Syed NH, Bordage S, Marshall J, Nimmo GA, Jenkins Gl, et al. Alternative splicing mediates responses of the Arabidopsis circadian clock to temperature changes. Plant Cell. 2012;24:961-81.

5. Khan S, Rowe SC, Harmon FG. Coordination of the maize transcriptome by a conserved circadian clock. BMC Plant Biol. 2010;10:126.

6. Legnaioli T, Cuevas J, Mas P. TOC1 functions as a molecular switch connecting the circadian clock with plant responses to drought. EMBO J. 2009;28:3745-57.

7. Covington MF, Maloof JN, Straume M, Kay SA, Harmer SL. Global transcriptome analysis reveals circadian regulation of key pathways in plant growth and development. Genome Biol. 2008;9:R130.

8. Harmer SL, Hogenesch JB, Straume M, Chang H, Han B, Zhu T, et al. Orchestrated transcription of key pathways in Arabidopsis by the circadian clock. Science. 2014;290:2110-3.

9. Schaffer R, Landgraf J, Accerbi M, Simon V, Larson M, Wisman E. Microarray analysis of diurnal and circadian-regulated genes in Arabidopsis. Plant Cell. 2001;13:113-23.

10. Pruneda-Paz $J$, Kay SA. An expanding universe of circadian networks in higher plants. Trends Plant Sci. 2010;15:259-65.

11. Covington MF, Harmer SL. The circadian clock regulates auxin signaling and responses in Arabidopsis. PLoS Biol. 2007;5:1773-84.

12. Mizuno T, Yamashino T. Comparative transcriptome of diurnally oscillating genes and hormone-responsive genes in Arabidopsis thaliana: insight into circadian clock-controlled daily responses to common ambient stresses in plants. Plant Cell Physiol. 2008;49:481-7.

13. Nováková M, Motyka V, Dobrev PI, Malbeck J, Gaudinová A, Vanková R. Diurnal variation of cytokinin, auxin, and abscisic acid levels in tobacco leaves. J Exp Bot. 2005;56:2877-83.

14. Carbonell-Bejerano P, Rodríguez V, Royo C, Hernáiz S, Moro-González LC, Torres-Viñals $\mathrm{M}$, et al. Circadian oscillatory transcriptional programs in grapevine ripening fruits. BMC Plant Biol. 2014;14:78.

15. Bieniawska Z, Espinoza C, Schlereth A, Sulpice R, Hincha DK, Hannah MA Disruption of the Arabidopsis circadian clock is responsible for extensive variation in the cold-responsive transcriptome. Plant Physiol. 2008;147:263-79.

16. Wilkins O, Waldron L, Nahal H, Provart NJ, Campbell MM. Genotype and time of day shape the Populus drought response. Plant J. 2009;60:703-15.

17. Wilkins O, Bräutigam K, Campbell MM. Time of day shapes Arabidopsis drought transcriptomes. Plant J. 2010;63:715-27.

18. Hudson KA. The circadian clock-controlled transcriptome of developing soybean seeds. Plant Genome J. 2010;3:3.

19. He C-Y, Zhang J-S, Chen S-Y. A soybean gene encoding a proline-rich protein is regulated by salicylic acid, an endogenous circadian rhythm, and by various stresses. Theor Appl Genet. 2002;104:1125-31.

20. Marcolino-Gomes J, Rodrigues FA, Fuganti-Pagliarini R, Bendix C, Nakayama TJ, Celaya B, et al. Diurnal oscillations of soybean circadian clock and drought responsive genes. PLoS One. 2014;9:e86402.

21. Oya T, Nepomuceno AL, Neumaier N, Farias JRB, Tobita S, Ito O. Drought tolerance characteristics of Brazilian soybean cultivars: evaluation and characterization of drought tolerance of various Brazilian soybean cultivars in the field. Plant Prod Sci. 2004;7:129-37.

22. Schmutz J, Cannon SB, Schlueter J, Ma J, Mitros T, Nelson W, et al. Genome sequence of the palaeopolyploid soybean. Nature. 2010;463:178-83.

23. Supek F, Bošnjak M, Škunca N, Šmuc T. REVIGO summarizes and visualizes long lists of gene ontology terms. PLoS One. 2011;6:e21800.

24. Hsu PY, Harmer SL. Circadian phase has profound effects on differential expression analysis. PLoS One. 2012;7:e49853.
25. Raeini-Sarjaz M. Circadian rhythm leaf movement of Phaseolus vulgaris and the role of calcium ions. Plant Signal Behav. 2011;6:962-7.

26. Frey A, Effroy D, Lefebvre $V$, Seo M, Perreau F, Berger A, et al. Epoxycarotenoid cleavage by NCED5 fine-tunes ABA accumulation and affects seed dormancy and drought tolerance with other NCED family members. Plant J. 2012;70:501-12.

27. Blanvillain R, Kim JH, Wu S, Lima A, Ow DW. OXIDATIVE STRESS 3 is a chromatin-associated factor involved in tolerance to heavy metals and oxidative stress. Plant J. 2009;57:654-65.

28. de Carvalho MHC. Drought stress and reactive oxygen species. Plant Signal Behav. 2008;3:156-65.

29. Borsani O, Díaz P, Agius MF, Valpuesta V, Monza J. Water stress generates an oxidative stress through the induction of a specific Cu/Zn superoxide dismutase in Lotus corniculatus leaves. Plant Sci. 2001;161:757-63.

30. Fraire-Velázquez S, Balderas-Hernández V. Abiotic stress in plants and metabolic responses. In: Vahdati K, Leslie C. Rijeka. Abiotic stress: plant responses and applications in agriculture. InTech is registered [(MBS): 040244148; (OIB): 71692491655]; Rijeka, Croatia. 2013: 25-48.

31. Dhaubhadel S, Browning KS, Gallie DR, Krishna P. Brassinosteroid functions to protect the translational machinery and heat-shock protein synthesis following thermal stress. Plant J. 2002;29:681-91.

32. Dhaubhadel S, Chaudhary S, Dobinson KF, Krishna P. Treatment with 24-epibrassinolide, a brassinosteroid, increases the basic thermotolerance of Brassica napus and tomato seedlings. Plant Mol Biol. 1999;40:333-42.

33. Manosalva PM, Davidson RM, Liu B, Zhu X, Hulbert SH, Leung H, et al. A germin-like protein gene family functions as a complex quantitative trait locus conferring broad-spectrum disease resistance in rice. Plant Physiol. 2009;149:286-96.

34. Wang T, Chen X, Zhu F, Li H, Li L, Yang Q, et al. Characterization of peanut germin-like proteins, AhGLPs, in plant development and defense. PLoS One. 2013;8:e61722.

35. Zimmermann G, Bäumlein H, Mock H-P, Himmelbach A, Schweizer P. The multigene family encoding germin-like proteins of barley: regulation and function in basal host resistance. Plant Physiol. 2006;142:181-92.

36. Lu M, Han Y-P, Gao J-G, Wang X-J, Li W-B. Identification and analysis of the germin-like gene family in soybean. BMC Genomics. 2010;11:620.

37. Membré N, Bernier F, Staiger D, Berna A. Arabidopsis thaliana germin-like proteins: common and specific features point to a variety of functions. Planta. 2000;211:345-54.

38. Staiger D, Apel K, Trepp G. The Atger3 promoter confers circadian clock-regulated transcription with peak expression at the beginning of the night. Plant Mol Biol. 1999;40:873-82.

39. Heintzen C, Fischer R, Melzer S, Kappeler S, Apel K, Staiger D. Circadian oscillations of a transcript encoding a germin-like protein that is associated with cell walls in young leaves of the long-day plant Sinapis alba. Plant Physiol. 1994;106:905-15.

40. Carter C, Graham RA, Thornburg RW. Arabidopsis thaliana contains a large family of germin-like proteins: characterization of CDNA and genomic sequences encoding 12 unique family members. Plant Mol Biol. 1998;38:929-43.

41. Hattori J, Boutilier KA, Campagne MML, Miki BL. A conserved BURP domain defines a novel group of plant proteins with unusual primary structures. Mol Gen Genet. 1998;259:424-8.

42. Van Son L, Tiedemann J, Rutten T, Hillmer S, Hinz G, Zank T, et al. The BURP domain protein AtUSPL1 of Arabidopsis thaliana is destined to the protein storage vacuoles, and overexpression of the cognate gene distorts seed development. Plant Mol Biol. 2009;71:319-29.

43. Ding $X$, Hou X, Xie K, Xiong L. Genome-wide identification of BURP domain-containing genes in rice reveals a gene family with diverse structures and responses to abiotic stresses. Planta. 2009;230:149-63.

44. Xu H, Li Y, Yan Y, Wang K, Gao Y, Hu Y. Genome-scale identification of soybean BURP-domain-containing genes and their expression under stress treatments. BMC Plant Biol. 2010;10:197.

45. Wang $H$, Zhou L, Fu Y, Cheung M-Y, Wong F-L, Phang T-H, et al. Expression of an apoplast-localized BURP-domain protein from soybean (GmRD22) enhances tolerance toward abiotic stress. Plant Cell Environ. 2012;35:1932-47.

46. Kant $\mathrm{P}$, Gordon $\mathrm{M}$, Kant S, Zolla G, Davydov $\mathrm{O}$, Heimer $\mathrm{YM}$, et al. Functionalgenomics-based identification of genes that regulate Arabidopsis responses to multiple abiotic stresses. Plant Cell Environ. 2008;31:697-714.

47. Urano K, Kurihara Y, Seki M, Shinozaki K. "Omics" analyses of regulatory networks in plant abiotic stress responses. Curr Opin Plant Biol. 2010;13:132-8. 
48. Spoel S, van Ooijen G. Circadian redox signaling in plant immunity and abiotic stress. Antioxid Redox Sign. 2013;20:1-46.

49. Fehr WR, Caviness C, Burmood D, Pernnigton J. Stage of development description for soybeans (Glycine max L, Merrill). Crop Sci. 1971;11:929-31.

50. Shukla A, Panchal H, Mishra M, Patel PR, Srivastava HS, Patel P, et al. Soil moisture estimation using gravimetric technique and FDR probe technique: a comparative analysis. Am Int J Res Formal, Appl Nat Sci. 2014;8:89-92.

51. Tariq MA, Kim HJ, Jejelowo O, Pourmand N. Whole-transcriptome RNAseq analysis from minute amount of total RNA. Nucleic Acids Res. 2011;39:e120

52. Li H, Durbin R. Fast and accurate short-read alignment with Burrows-Wheeler transform. Bioinformatics. 2009;25:1754-60.

53. Robinson MD, Mccarthy DJ, Smyth GK. edgeR: a Bioconductor package for differential expression analysis of digital gene expression data. Bioinformatics. 2010;26:139-40.

54. Benjamini $Y$, Rochberg $Y$. Controlling the false discovery rate: a practical and powerful approach to multiple testing. J R Stat Soc Series B Methodol. 1995:57:289-300

55. Du Z, Zhou X, Ling Y, Zhang Z, Su Z. AgriGO: a GO analysis toolkit for the agricultural community. Nucleic Acids Res. 2010;38:64-70.

56. Hughes ME, Hogenesch JB, Kornacker K. JTK_CYCLE: an efficient nonparametric algorithm for detecting rhythmic components in genome-scale datasets. J Biol Rhythm. 2010;25:372-80.

57. Livak KJ, Schmittgen TD. Analysis of relative gene expression data using real-time quantitative PCR and the 2(-Delta Delta C(T)) method. Methods. 2001;25:402-8

58. Jian B, Liu B, Bi Y, Hou W, Wu C, Han T. Validation of internal control for gene expression study in soybean by quantitative real-time PCR. BMC Mol Biol. 2008:9:59.

59. Stolf-Moreira R, Lemos EGM, Abdelnoor RV, Beneventi MA, Rolla AAP, Pereira SS, et al. Identification of reference genes for expression analysis by real time. Pesqui Agropec Bras. 2011;46:58-65.

\section{Submit your next manuscript to BioMed Central and take full advantage of:}

- Convenient online submission

- Thorough peer review

- No space constraints or color figure charges

- Immediate publication on acceptance

- Inclusion in PubMed, CAS, Scopus and Google Scholar

- Research which is freely available for redistribution 